\title{
Reinhard Jung
}

Reverse Engineering konzeptioneller Datenschemata 
Reinhard Jung

\section{Reverse Engineering konzeptioneller Dafenschemata}

Vorgehensweisen und Rekonstruierbarkeit für Cobol-Programme 
Die Deutsche Bibliothek - CIP-Einheitsaufnahme

Jung, Reinhard:

Reverse-Engineering konzeptioneller Datenschemata : Vorgehensweisen und Rekonstruierbarkeit für Cobol-Programme / Reinhard Jung. -

(DUV : Wirtschaftsinformatik)

Zugl.: Münster (Westfalen), Univ., Diss., 1998

ISBN 978-3-8244-2109-1 ISBN 978-3-663-08926-1 (eBook)

DOI 10.1007/978-3-663-08926-1

Alle Rechte vorbehalten

(c) Springer Fachmedien Wiesbaden 1998

Ursprünglich erschienen bei Deutscher Universitäts-Verlag GmbH, Wiesbaden, 1998

Lektorat: Monika Mülhausen

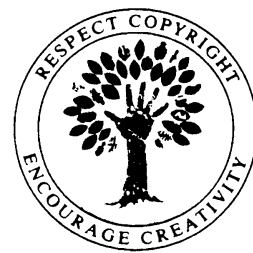

Das Werk einschließlich aller seiner Teile ist urheberrechtlich geschützt. Jede Verwertung außerhalb der engen Grenzen des Urheberrechtsgesetzes ist ohne Zustimmung des Verlages unzulässig und strafbar. Das gilt insbesondere für Vervielfältigungen, Übersetzungen, Mikroverfilmungen und die Einspeicherung und Verarbeitung in elektronischen Systemen.

http://www.duv.de

ISBN 978-3-8244-2109-1 


\section{Vorwort}

Insbesondere große Unternehmen betreiben häufig ein Vielzahl von Anwendungssystemen zur Transaktionsverarbeitung, die eine lange Entwicklungsgeschichte besitzen. Die meisten dieser Systeme basieren auf veralteter Softwaretechnik: Cobol-Programme mit proprietärer Datenverwaltung. Neben dem inaktuellen Technologiestand, den die Altsysteme aufweisen, fehlt häufig auch eine Dokumentation des gegenwärtigen Zustands der Software, so daß die Wartung immense Kosten verursacht.

Parallel zur Altsystemproblematik wird in jüngster Zeit sehr intensiv der Einsatz von betriebswirtschaftlichen Standardsoftwarepaketen diskutiert, propagiert und auch praktiziert. Vielfach werden die Wartungsproblematik, das Jahr-2000-Problem oder die Einführung des EURO als Argumente für die Ersetzung der Altsysteme herangezogen. Derartige Ersatzentscheidungen sind allerdings ökonomisch wenig fundiert. Die vorliegende Arbeit versucht, in diesem Bereich einen Beitrag zu einer solideren Entscheidungsgrundlage zu leisten. Es ist zwar nicht möglich, das Ersatzzeitpunktproblem von Software vollständig und exakt zu lösen, es sind aber doch interessante Erkenntnisse zu gewinnen in diesem von der Informatik und auch von der Betriebswirtschaftslehre etwas stiefmütterlich behandelten Gebiet.

Ein Promotionsprojekt ist typischerweise nicht nur für den Autor, sondern auch für sein gesamtes Umfeld eine "harte Prüfung". Es ist kaum möglich, an dieser Stelle allen zu danken, die es verdient hätten. Deshalb seien hier nur die besonders in Anspruch genommenen Personen erwähnt.

Meinem Doktorvater, Herrn Professor Dr. Karl Kurbel, danke ich nicht nur für seinen Beitrag zum Gelingen meiner Dissertation, sondern auch für die Vermittlung von Know-how, das sich im wissenschaftlichen "Berufsalltag" als sehr nützlich erwiesen hat. Herrn Professor Dr. Jörg Becker, meinem Zweitgutachter, danke ich für die Übernahme des Koreferats und die eingebrachte konstruktive Kritik.

Auch meinem jetzigen Chef, Herrn Professor Dr. Robert Winter, schulde ich Dank für fruchtbare inhaltliche Diskussionen und den großzügig gewährten Freiraum zur endgültigen Fertigstellung des vorliegenden Buchs. Ferner danke ich meinen Eltern, die "mein Projekt" selbst in 
schwierigen Zeiten in vielfältiger Weise sehr engagiert unterstützt haben. Zum guten Schluß danke ich meiner Freundin, Frau Dr. Ulrike Baumöl, besonders herzlich, da sie sicherlich die wesentlichen "Unbilden" meiner Promotionszeit miterlebt hat. Trotz starker beruflicher Inanspruchnahme und einem eigenen Promotionsprojekt hat sie immer viel Verständnis aufgebracht und mich uneingeschränkt unterstützt.

Reinhard Jung 


\section{Inhaltsverzeichnis}

$\begin{array}{ll}\text { Abkürzungsverzeichnis } & \text { XI }\end{array}$

1 Einleitung 1

1.1 Problembereich und Einordnung der Arbeit 1

1.2 Zielsetzung der Arbeit 3

1.3 Aufbau der Arbeit 5

2 Terminologische und technische Grundlagen des Software Reengineering $\quad 7$

2.1 Entstehung und Begrifflichkeit des Software Engineering 7

2.2 Software Reengineering und Reverse Engineering $\quad 10$

$\begin{array}{ll}2.2 .1 \text { Begriffsbestimmung } & 10\end{array}$

2.2.2 Einordnung des Software Reengineering in die Softwarewartung und -wiederverwendung $\quad 14$

$\begin{array}{ll}\text { 2.2.2.1 Wartung } & 14\end{array}$

2.2.2.2 Wiederverwendung 16

$\begin{array}{ll}\text { 2.2.3 Reengineering-Strategien und -Ziele } & 17\end{array}$

$\begin{array}{ll}2.2 .4 & \text { Reengineering-Werkzeuge } \\ & 21\end{array}$

2.3 Altsystem-basierte Softwareentwicklung 24

2.3.1 Beeinflussung der verwendeten Vorgehensweise durch die
Einbeziehung eines Softwarealtsystems

2.3.2 Wiederverwendung konzeptioneller Datenschemata im Rahmen einer Neuentwicklung

2.3.3 Besonderheiten des Werkzeugeinsatzes bei altsystem-basierter $\begin{array}{ll}\text { Softwareentwicklung } & 30\end{array}$

3 Software Reengineering aus betriebswirtschaftlicher Sicht 33

3.1 Wirtschaftlichkeit im Kontext von Software 33

$\begin{array}{lll}3.1 .1 & \text { Investitionen in Anwendungssoftware } & 34\end{array}$

3.1.2 Strukturierung der Wirtschaftlichkeitsbetrachtung anhand grober
Softwarelebenszyklus-Phasen

3.2 Wirtschaftlichkeit der Softwarebereitstellung 42

3.3 Wirtschaftlichkeit der Softwarenutzung 44

3.4 Zusammenfassung $\quad 46$ 
4 Messungen als Voraussetzung und Ausgangspunkt von ReengineeringMaßnahmen

4.1 Bedeutung der Softwarequalität im Kontext des Reverse Engineering $\quad 47$

4.1.1 Berücksichtigung der Rekonstruierbarkeit in bekannten $\begin{array}{ll}\text { Softwarequalitätsmodellen } & 47\end{array}$

$\begin{array}{ll}\text { 4.1.2 Softwarequalität von Altsystemen } & 52\end{array}$

$\begin{array}{lll}4.2 & \text { Software-Metrie } & 53\end{array}$

4.2.1 Abbildung zwischen empirischen und numerischen Relationensystemen 54

$\begin{array}{ll}\text { 4.2.2 Meßskalen } & 56\end{array}$

$\begin{array}{ll}\text { 4.2.3 Klassifikation von Metriken } & 58\end{array}$

$\begin{array}{ll}\text { 4.2.4 Gütekriterien für Metriken } & 59\end{array}$

$\begin{array}{lll}4.3 & \text { Kosten- und Aufwandschätzverfahren } & 61\end{array}$

4.3.1 Berücksichtigung des Reengineering in bekannten Kosten- und $\begin{array}{ll}\text { Aufwandschätzverfahren } & 61\end{array}$

4.3.2 Qualität von Kosten- und Aufwandsschätzungen 69

$\begin{array}{lll}4.4 & \text { Zusammenfassung } & 70\end{array}$

5 Grundlagen der Datenschema-Rekonstruktion und der Beurteilung der Datenschema-Rekonstruierbarkeit

5.1 Struktur der Untersuchung zu den unterschiedlichen

Datenhaltungskonzepten

5.2 Auswahl und Darstellung der verwendeten Beschreibungsmethoden 73

$\begin{array}{lll}\text { 5.2.1 } & \text { Erweitertes ER-Modell } & 74\end{array}$

5.2.2 Repräsentation des physischen Schemas bei einer Cobol-

Dateiverwaltung

5.2.2.1 Rekonstruktion konzeptioneller Schemata anhand von CobolDateistrukturen in der Literatur $\quad 78$

$\begin{array}{ll}\text { 5.2.2.2 Erweiterte Datenstrukturdiagramme } & 81\end{array}$

5.3 Grundsätzlicher Ansatz zur Beurteilung der Rekonstruierbarkeit des konzeptionellen Datenschemas

6 Rekonstruktion des ER-Schemas auf Basis einer Dateiverwaltung 91

6.1 Nutzung von Relationen als logische Dateien 91

6.2 Rekonstruktion von Ausgangs-EDSDs 94

6.2.1 Rekonstruktion von Ausgangs-EDSDs anhand eines relationalen
Schemas

6.2.2 Rekonstruktion von Ausgangs-EDSDs anhand spezifischer Konstrukte der Cobol-Dateiverwaltung 95

6.2.2.1 Erstellung von Ausgangs-EDSDs anhand der File Section 96 
6.4 Ermittlung der Semantik der EDSD-Komponenten

6.5 Restrukturierung des EDSDs

6.6 Transformation der EDSDs in ein „First cut"-Schema

6.7 Rekonstruktion von Schlüsselinformationen und Beziehungstypen

6.8 Entsprechungen zwischen Konstrukten der Dateiverwaltung und des ERModells

6.9 Meßvorschriften und Indikatoren zur Beurteilung eines Altsystems

6.9.2 Metriken zur Beurteilung der Quelltextkomplexität

6.10 Diskussion der grundsätzlichen Eignung für die Rekonstruktion des konzeptionellen Datenschemas

7.1 Konstruktionselemente eines relationalen Schemas

7.2 Grundsätzlicher Ablauf der Rekonstruktion

7.3 Die Vorgehensweise von Markowitz und Makowsky

7.3.2 Transformation des relationalen Schemas in ein ER-Schema

7.4 Anreicherung des ER-Schemas um semantische Informationen

7.5 Entsprechungen zwischen Konstrukten des relationalen Modells und des ER-Modells

7.6 Meßvorschriften und Indikatoren zur Beurteilung eines Altsystems

7.7 Diskussion der grundsätzlichen Eignung für die Rekonstruktion des konzeptionellen Datenschemas 
8 Rekonstruktion des ER-Schemas auf Basis eines netzwerkartigen oder hierarchischen Schemas

$\begin{array}{lll}\text { 8.1 Netzwerkartige Schemata } & 139\end{array}$

$\begin{array}{ll}\text { 8.1.1 Konstruktionselemente eines netzwerkartigen Schemas } & 139\end{array}$

$\begin{array}{ll}\text { 8.1.2 Rekonstruktion auf Basis eines netzwerkartigen Schemas } & 141\end{array}$

8.1.2.1 Die Vorgehensweise von Batini et al. 142

8.1.2.2 Die Vorgehensweise von Michely und Scheer 143

8.1.2.3 Konstruktion einer geeigneten Vorgehensweise 146

8.2 Hierarchische Schemata 151

8.2.1 Konstruktionselemente eines hierarchischen Schemas 151

8.2.2 Rekonstruktion auf Basis eines hierarchischen Schemas 153

8.2.2.1 Die Vorgehensweise von Batini et al. 153

8.2.2.2 Die Vorgehensweise von Dumpala und Arora 154

8.2.2.3 Konstruktion einer geeigneten Vorgehensweise 155

8.3 Entsprechungen zwischen Konstrukten des netzwerkartigen bzw. hierarchischen Datenbankmodells und des ER-Modells

8.4 Meßvorschriften und Indikatoren zur Beurteilung eines Altsystems

8.5 Diskussion der grundsätzlichen Eignung für die Rekonstruktion des konzeptionellen Datenschemas

$\begin{array}{lll}9.1 \text { Zusammenfassung der Ergebnisse } & 161\end{array}$

9.2 Übertragbarkeit der Ergebnisse zur Lösung weiterer Aufgabenstellungen 163

$\begin{array}{lll}9.3 & \text { Ausblick } & 164\end{array}$ 


\section{Abkürzungsverzeichnis}

ACM Association for Computing Machinery

ACT Annual change traffic

ADABAS Adaptiertes Datenbanksystem

ANSI American National Standards Institute

ASLOC Adapted source lines of Code

BPR Business process reengineering

CARE Computer aided reengineering

CASE Computer aided software engineering

COCOMO Constructive cost model

Codasyl Conference on Data Systems Languages

DBD Data base description

DBTG Data base task group

DIN Deutsches Institut für Normung

DDL Data definition language

DML Data manipulation language

DSD Datenstrukturdiagramm (Data structure diagram)

ECR-Modell Entity-Category-Relationship-Modell

EDSD erweitertes Datenstrukturdiagramm

ERCM Entity relationship conceptual model

ERDM Entity relationship data model

ESLOC Equivalent source lines of code

FD File description

HMD Handbuch der modernen Datenverarbeitung (Untertitel: Theorie und Praxis der Wirtschaftsinformatik)

I-CASE Integrated computer aided software engineering 
i.d.R

in der Regel

i.S.v. im Sinne von

IBM International business machines

IDMS Integrated database management system

IEEE The Institute of Electrical and Electronics Engineers

IMS Information management system

ISO International Organization for Standards

KDSI Produktumfang gemessen in Thousands of delivered source instructions (1000 Quelltext-Anweisungen)

MIS

Management information systems

$\mathrm{MM}_{\mathrm{AM}}$ Jährlicher Wartungsaufwand

$\mathrm{MM}_{\mathrm{DEV}}$

Entwicklungsaufwand

o.O. ohne Ort

PCB Program communication block

$\mathrm{RD}$ Report description

RDBMS Relationales Datenbankmanagementsystem

SD Sort file description

SLOC Source lines of code

SQL Structured query language

TDEV Development schedule (Entwicklungsdauer)

VRT Visual Reengineering Toolset (von McCabe and Associates)

ZfB Zeitschrift für Betriebswirtschaft 